\title{
Special SeCtion Guest EDITORIAL
}

\section{Optics in Breast Cancer}

Breast cancer is the leading cause of cancer-related death in women in the western world. Conventional breast cancer screening with x-ray mammography has been a clear benefit if applied population-wide, and under stringent quality assessment. However, even under optimal conditions, mortality reductions are not higher than $30 \%$, and even this benefit has not been unequivocally accepted. While breast cancer mortality appears to decline in those countries where nation-wide screening is provided, still almost 80,000 women in Europe and 40,000 in the U.S. die from the disease every year.

In addition, the efficiency of x-ray mammography is inferior in premenopausal women, while our extended knowledge about genetic predisposition to breast cancer now confronts young women with the knowledge of an increased breast cancer risk. Given this, providing a suitable early detection procedure or acceptable prevention method is needed.

Digital mammography may improve the diagnostic accuracy of mammography to a limited extent but will not provide a breakthrough in terms of sensitivity nor specificity, and will likely not provide the needed tool for screening younger women or those with radio-opaque breasts.

Breast sonography is an established adjunct to mammography. Its early detection potential was validated in the past two decades, but so far, ultrasound examination alone was determined unsuitable for breast cancer screening.

Contrast enhanced magnetic resonance imaging (MRI) of the breast is actually the most promising diagnostic modality to improve breast cancer detection, especially in young women with dense breast parenchyma. Recent clinical studies have underlined this potential in women with a genetically determined predisposition for the development of breast cancer. Earlier specificity disadvantages may be overcome with appropriate MR-guided biopsy techniques. However, MRI still requires the use of contrast agents, is technically highly demanding, and leads to a larger number of biopsies.

Finally, breast tissue biopsy itself is typically taken as the deciding factor in the diagnosis of disease, yet errors due to sampling volume and region error still occur.

In this context, optical methods are appealing due to their potential for increased sensitivity and specificity when used in certain settings. Optical techniques have been investigated to study and characterize breast cancer for almost a century, yet today the methods proposed are distinctly different in terms of the technology used and algorithms applied. Because breast cancer diagnosis has become such a successful test bed for new technology, it provides a suitable disease site for developing improved tools for early diagnosis, which may either impact management by improving the sensitivity or the specificity, or provide similar information to conventional methods but without the need for invasive or ionizing radiation procedures. Optical technology and algorithms have continuously progressed, and currently there are many clinical trials ongoing with a wide variety of specific goals. This special section is devoted to a range of diagnostic optical methods currently being examined for breast cancer diagnosis.

In the past decade it has become apparent that characterization of tissues based upon the scattered spectrum provides a unique set of information, which is rich in both the chromophores present and also in the size and density of scattering features. The first two papers in this special section, by Johnson et al. and van Veen et al., explore ways in which intrasurgical measurements can be taken directly from tumor tissue in order to measure these features. Extraction of scattering and absorber features provides fundamental insight about the feasibility of using this type of measurement as a diagnostic tool, either used invasively as with needle biopsy or during a surgical procedure.

Noninvasive near-infrared (NIR) measurements can be used to extract quantitatively useful parameters for hemoglobin, water, oxygen saturation, lipids, etc., as well as scatterer information. The range of technologies used in this noninvasive imaging approach still varies widely, and as clinical studies progress the particular niche areas of radiology where this technology may contribute will become more apparent. The next three papers, by Spinelli et al., Pifferi et al., and Heffer et al., examine ways to develop and analyze these types of measurements in a way that provides the maximal pertinent information. The spectral interpretation of NIR measurements is simple in essence, yet complex in the accuracy and details when applied to a deformable and heterogeneous organ such as the breast. Each of these papers covers a different aspect of this complicated problem.

The last two papers in the special section focus around challenges in the spatial formation of images. The first paper, by Srinivasan et al., examines new methods for tomographic image formation of heterogeneities, based upon the challenging ill-posed problem of recovering the chromophore and scatterer images from multiple source-detector projection imaging. The final paper, by Manohar et al., introduces a newly emerging modality using photoacoustic measurement to form images of the interior of bulk tissue. This modality presents the possibility of imaging through thicker tissues due to the higher penetration of ultrasound signals, and yet retaining the high intrinsic contrast of NIR absorption.

Taken together these papers cover a very broad range of areas where optical systems are being developed and tested for their utility in diagnosis of breast cancer.

Sergio Fantini, Ph.D., Tufts University, Medford, Massachusetts

K. Thomas Moesta, M.D., Charité, Berlin, Germany

Brian W. Pogue, Ph.D., Dartmouth College, Hanover, New Hampshire 